\title{
La retroalimentación en la resolución de problemas fraccionarios, con alumnos de quinto grado de primaria, mediante el uso de una aplicación
}

\author{
Feedback on answers to questions involving fractions with fifth \\ grade elementary school students through the use of an app
}

DOI: https://doi.org/10.32870/dse.v0i22.830

\section{Uriel López Marques*}

\begin{abstract}
Resumen
El presente estudio se llevó a cabo con cuatro estudiantes de quinto grado del municipio de Yahualica, Jalisco, en el contexto de la pandemia causada por el COVID-19. En este trabajo se asume que la retroalimentación es una de las más poderosas herramientas para el aprendizaje y el logro educativo. La investigación parte de las concepciones de retroalimentación desde una perspectiva de Aprendizaje y Enseñanza Visible propuesta por Hattie (Hattie, Gan, 2011), y basada en el modelo de Retroalimentación de Hattie y Timperley (2007). El horizonte metodológico empleado para este estudio corresponde a una perspectiva cualitativa (Creswell, 2012), mediante el estudio de caso (Stake, 1999). El método de investigación utilizado fue la observación participante (Creswell, 2012), así como la entrevista semiestructurada (Kvale, 2011) para recabar datos cualitativos de los estudiantes, quienes interactuaron con una aplicación para la resolución de problemas fraccionarios. Para el anális de datos se utilizó el doble ciclo de codificación propuesto por Saldaña (2009). Como resultado de la investigación se concluyó que los estudiantes buscan la retroalimentación para encontrar procedimientos de mejora, y para que esta sea utilizada es necesario que exista un ambiente adecuado del aprendizaje donde se vea al error como una oportunidad de aprender.
\end{abstract}

Palabras claves: retroalimentación - ambientes de aprendizaje - aplicación - fracciones - aprendizaje.

\begin{abstract}
This study was carried out with four fifth grade students from Yahualica, Jalisco, in the context of the pandemic caused by COVID-19. Feedback is assumed in this paper to be one of the most powerful tools for learning and educational achievement. The research is based on the concept of feedback as a perspective of Visible Learning and Teaching proposed by Hattie (Hattie, Gan, 2011), and Hattie \& Timperley's Feedback model (2007). The methodological horizon used for this study corresponds to a qualitative perspective (Creswell, 2012) using a case study (Stake, 1999). The research method used participant obser-
\end{abstract}

\footnotetext{
* Estudiante del Doctorado en Investigación Educativa Aplicada. Instituto Superior de Docencia para el Magisterio (ISIDM). Asesor Técnico Pedagógico para la Secretaría de Educación Pública. México. uriellopezmarques@gmail.com
} 
vation (Creswell, 2012), as well as a semi-structured interview (Kvale, 2011) to collect qualitative data from the students, who interacted with an application for solving fractional problems. For the data analysis, the double coding cycle proposed by Saldaña (2009) was used. As a result of our research, we concluded that students seek feedback to find improvement procedures, and for it to be used there must be an adequate learning environment where error is seen as an opportunity to learn.

Keywords: feedback - learning environment - application - fractions - learning.

\section{Introducción}

La pandemia causada por el COVID-19 modificó en forma sustantiva la manera de ser y de actuar de todos los ciudadanos del planeta. Una de las áreas que, sin duda, tuvo que reinventarse fue la educación y todos sus componentes, incluyendo la investigación educativa.

Un ejemplo claro fue la transformación que sufrió el presente trabajo: originalmente se pensó utilizar una perspectiva mixta a través del diseño incrustado (Creswell, 2012), empleando un diseño cuasi-experimental en una primaria ubicada en el municipio de Zapopan, Jalisco, y se iba a trabajar con dos grupos de quinto grado. No obstante, luego de declararse la contingencia y ante la incertidumbre acerca de fechas precisas para el regreso a las actividades presenciales, así como las trasformaciones que surgirían ante la nueva normalidad, se decidió modificar la manera de realizar la investigación y aprovechar los nuevos escenarios de educación a distancia, así como la disponibilidad de los estudiantes y padres de familia para dar respuesta a las interrogantes que se planteaban en la investigación.

\section{Planteamiento del problema}

Es importante mencionar que si bien la finalidad de este trabajo es reconocer la manera en que los estudiantes emplean la retroalimentación formativa para aprender, además de problematizar ese concepto dada la naturaleza del estudio, se seleccionó un contenido complejo para los estudiantes: la resolución de problemas fraccionarios, a fin de reconocer la manera en que los alumnos utilizan la retroalimentación para aprenderlo.

\section{Preguntas y propósitos de investigación}

Debido a las reducidas oportunidades que tienen los educandos para recibir retroalimentación formativa dadas las dificultades de los docentes para emplearla como un elemento de mejora de los estudiantes, se consideró importante construir la aplicación Retromath, que proporciona a los estudiantes la oportunidad de retroalimentarlos para mejorar su aprendizaje.

La presente investigación plantea que la utilización de las Tecnologías de la Información y la Comunicación (TIC), mediante el uso de la aplicación Retromath, pudiera ser una opción que

Diólo@os sobre Educación año 12 | número 22 | enero-junio 2021 | ISSN 2007-2171 
ayude a los docentes en su tarea de brindar retroalimentación y, sobre todo, que dé a los estudiantes la oportunidad de recibirla para mejorar sus procesos de aprendizaje.

De lo anteriormente expuesto surgen las siguientes preguntas y propósitos de investigación que orientan este trabajo:

1. ¿Cómo entienden los estudiantes la retroalimentación? ¿Cuáles son sus percepciones de recibirla?

2. ¿Cómo utilizan la retroalimentación los estudiantes?

3. ¿De qué manera la retroalimentación favorece los resultados de aprendizaje de los estudiantes?

4. ¿A qué dificultades se enfrentan los estudiantes para emplear la retroalimentación?

Las preguntas anteriores atenderán a los siguientes propósitos:

\section{Propósito general:}

Comprender la manera en que la retroalimentación, brindada mediante la aplicación Retromath, favorece el aprendizaje del contenido: "resolución de problemas fraccionarios" en quinto grado de primaria.

Propósitos específicos:

-Analizar la manera en que los alumnos utilizan la retroalimentación proporcionada por la aplicación Retromath para la resolución de problemas fraccionarios.

-Reconocer las percepciones que tienen los estudiantes respecto de recibir retroalimentación brindada por la aplicación Retromath.

-Identificar los elementos que dificultan a los estudientes el uso de la retroalimentación.

\section{Situación problemática 1: la retroalimentación}

De acuerdo con Hattie y Timperley (2007), la retroalimentación es una de las más importantes influencias para el aprendizaje y la mejora del desempeño, incluso es considerada una de las cinco mejores estrategias que favorecen el logro de los aprendizajes. No obstante, a pesar de sus beneficios, también se identifican algunos factores que limitan su adecuada puesta en práctica en el aula, entre ellos: la conceptualización del profesor, relacionada con su formación docente; elementos contextuales como la gran cantidad de estudiantes en el salón de clases y sus heterogéneos niveles de aprendizaje; la exigencia de estudiantes y padres de familia de recibir una retroalimentación numérica, proveniente de una visión de la evaluación como exclusivamente sumativa, que aporta poco en la mejora, en contraste con la evaluación formativa. A continuación se describen las limitantes mencionadas. 


\section{Limitada formación docente y prácticas de retroalimentación deficientes}

En lo que respecta a la formación del profesorado en materia de evaluación educativa es importante señalar que, de acuerdo con Martínez Rizo (2013: 131) "solo la mitad de estados americanos exigen formación en evaluación como requisito para la certificación de los docentes y pocos programas de formación de maestros exigen que los alumnos lleven un curso sobre evaluación".

De lo anterior, puede inferirse la limitada formación docente orientada hacia la puesta en práctica de instrumentos para la evaluación de los aprendizajes, así como para brindar retroalimentación mediante la información recuperada en los mismos.

Por otro lado, el mismo Martínez Rizo (2013) reconoce que la formación inicial de los profesores mexicanos ha sido débil en materia de evaluación y los programas de actualización para los docentes en servicio también han descuidado este aspecto.

Respecto a lo anterior, Ravela, Picaroni y Loureiro (2017: 144) apuntan que la mayoría de las prácticas de retroalimentación están enfocadas exclusivamente a la corrección de errores. Además, luego de un estudio sistemático en diversos países de América Latina, encontraron que "la devolución por escrito se limita a marcas de correcto/incorrecto o a la asignación de puntos y calificaciones. Apenas $4 \%$ del total de propuestas [...] de Colombia y 3\% en Uruguay, tenían algún tipo de comentario escrito del profesor".

Por otro lado, una investigación con profesores de primaria, realizada entre 2012 y 2013 por la Universidad de Aguascalientes (México), arrojó que la retroalimentación proporcionada por los docentes consistía en "informar sobre el resultado de la calificación, en la forma de una nota [...] acompañada de elogios o amonestaciones [...] sin orientación sobre cómo conseguirlo" (Mercado, Martínez Rizo, 2014, en Ravela, 2017: 145).

Otra dificultad encontrada por Carless (2016) fue que la retroalimentación a los estudiantes, por lo general, era proporcionada al grueso de la clase, lo que hacía que creyeran que los comentarios no estaban dirigidos a cada uno en lo personal, no los ayudaba a mejorar su proceso de aprendizaje y carecían de pautas para continuar. Además, Goldstein (2006, en MacDonald, 2015) identificó que los alumnos tenían dificultades para aplicar la retroalimentación dada y reconocer cuáles serían los pasos a seguir en su proceso.

\section{Cantidad excesiva de alumnos en el aula de clases}

Otro elemento que complejiza el brindar retroalimentación a los estudiantes es la gran carga de trabajo que implica. Si se considera que los grupos de nivel primaria en la Zona Metropolitana de Guadalajara son de 30 a 35 estudiantes en promedio, y que cada uno posee niveles distintos de aprendizaje, resulta difícil para el docente retroalimentarlos en lo individual, para que reflexionen en sus producciones y puedan mejorar de manera constante.

Diólo pos 
Gibbs y Simpson (2014 en García-Jiménez, 2015: 15) coinciden con las ideas anteriores y señalan algunos elementos que obstaculizan las prácticas de retroalimentación en las aulas: "los profesores se encuentran sometidos a enormes presiones de tiempo, debido al fenómeno de la 'modularización' de las materias, a un mayor número de alumnos por clase y a una mayor diversidad de alumnado".

\section{Concepciones de la comunidad escolar respecto a la evaluación y la retroalimentación} Otro factor que dificulta brindar retroalimentación es la presión de alumnos y padres de familia por observar una calificación numérica en las producciones de los estudiantes.

De acuerdo con Martínez Rizo (2013), los profesores se sienten preocupados por las demandas de calificaciones de los padres de familia y la competencia entre los alumnos. No obstante, Wiliam (2011, en MacDonald, 2015) señala que brindar comentarios por escrito en lugar de calificaciones trae mejores resultados de aprendizaje. Asimismo, Butler (1998), en uno de sus estudios apoya las ideas anteriores; señala que la retroalimentación a manera de calificaciones no causa ningún beneficio para el aprendizaje de los alumnos, y respalda la idea antes mencionada de Wiliam (2011, en MacDonald, 2015), asegurar que al brindar retroalimentación mediante comentarios, aunado a colocar calificaciones en ellos, trae como resultado un menor desempeño de los alumnos.

En lo que respecta a los alumnos, Wiliam (2011, en Ravela, Picaroni, Loureiro, 2017: 230) señala que: "lo primero que miran los estudiantes cuando se les entrega un trabajo o un boletín, son sus notas. Y lo segundo, inmediatamente después, son las notas de sus compañeros". Respecto de esto, el mismo autor realiza una valoración en la que expone la poca utilidad de las notas, cuyo único fin se convierte en instrumento de comparación y clasificación.

Lamentablemente, estas situaciones traen consigo un ambiente que hace importantes las recompensas y la competencia, donde hay ganadores y perdedores. No obstante, para que haya aprendizaje es necesario dotar a los alumnos de retroalimentación en un ambiente donde el error sea bienvenido, y en el que todos pueden cometerlo sin que implique una descalificación (Souter, 2009).

\section{Situación problema 2: Las fracciones}

Para comprender la manera en que los estudiantes utilizan la retroalimentación, objeto de estudio de esta investigación, como una herramienta para aprender, se eligió un contenido en el que se obtienen bajos resultados de aprovechamiento: la "resolución de problemas que impliquen sumar o restar fracciones cuyos denominadores son múltiplos uno de otro" (SEP, 2011: 76).

Es importante destacar que no se trata de la resolución de problemas fraccionarios en sí, sino de la forma de retroalimentación y la manera en que la utilizan los estudiantes para aprender; no obstante, durante el primer acercamiento a la institución en que se llevaría a cabo, una 
de las preocupaciones de la directora y el colectivo docente recaía en las dificultades de los estudiantes en el aprendizaje de las fracciones, ya que los alumnos de quinto grado obtuvieron un promedio de $23.14 \%$ de aciertos en los reactivos correspondientes en la Evaluación Diagnóstica propuesta por la Secretaría de Educación, durante el mes de septiembre de 2019.

Por ello se optó por coadyuvar a resolver el problema identificado en esta escuela mediante el uso de retroalimentación formativa. Además, para conocer el panorama de esta problemática en distintas latitudes, se realizó una investigación bibliográfica de la que se da cuenta a continuación.

En un estudio realizado por Hannula (2003, en Cortina, Zúñiga, Visnovska, 2013) en el que se aplicaron 1,154 pruebas a estudiantes finlandeses de quinto grado, se encontró que $46 \%$ de ellos no fueron capaces de sombrear " $3 / 4$ " de una barra dividida en ocho partes iguales. Estudios análogos se hicieron en Australia por Gould, Outhred y Mitchelmore (2006, en Cortina, Zúñiga, Visnovska, 2013) obteniéndose resultados similares.

Asimismo, Cortina, Cardoso y Zúñiga (2012, en Cortina, Zúñiga, Visnovska, 2013) encontraron que $20 \%$ de los estudiantes de sexto grado de 13 escuelas mexicanas no asociaron la inscripción " $1 / 2$ " con la noción de mitad.

Además, mediante el Plan Nacional para la Evaluación de los Aprendizajes (PLANEA) 2015 para sexto grado, se obtuvo como resultado que solo cerca de $7 \%$ de los estudiantes fueron capaces de resolver problemas que involucraban números naturales, decimales y fraccionarios (González-Retana, Eudave-Muñoz, 2018).

Finalmente, solo $18 \%$ de los estudiantes de sexto grado que contestaron los Exámenes de Calidad y Logro Educativo (Excale) en 2009, pudieron resolver problemas de fracciones que relacionan dos números que representan la parte y el todo (González-Retana, Eudave-Muñoz, 2018).

Para concluir este apartado, es importante decir que existen diversos trabajos en que se analiza cómo la retroalimentación mediante dispositivos tecnológicos podría beneficiar ciertos procesos matemáticos (Huey-Min, 2017; Delacruz, 2018; Barrón-Estrada, Zatarian-Cabada, Hernández-Pérez, 2014).

Por ejemplo, Huey-Min (2017) propuso la utilización de cuestionarios en computadora para favorecer el contenido de resolución de problemas fraccionarios en estudiantes de primaria en Taiwan. Dichos cuestionarios se presentaban en dos modalidades, el primero con retroalimentación dinámica y adaptativa con instrucción individualizada, y el segundo solo con instrucción individualizada.

La retroalimentación dinámica adaptativa consistía en que a través del algoritmo que poseía el programa informático podía administrar a los estudiantes preguntas acordes con su Zona de Desarrollo Próximo (ZDP). En ambos, la instrucción individualizada les brindaba ayudas para resolver problemas matemáticos que se les presentaban.

Diálo@os año 12 | número 22 | enero-junio 2021 | ISSN 2007-2171 
Para evaluar el estudio se comparó el desempeño de tres grupos (uno para cada uno de los tipos de cuestionario y el tercero, que recibió instrucción sin hacer uso de ningún programa informático, solo la proporcionada por su profesor en el salón de clases), mediante un diseño pretest/postest para grupos no equivalentes. Los resultados mostraron que el grupo en el que se administró la retroalimentación dinámica y adaptativa superó a los otros dos.

\section{Marco teórico conceptual}

\section{La retroalimentación}

De acuerdo con García-Jiménez (2015: 8) la retroalimentación es "aquella información que se utiliza para reducir la diferencia entre los resultados de aprendizaje obtenidos por el estudiante y los resultados de aprendizaje esperados".

Este concepto coincide con el de Hattie y Timperley (2007), quienes afirman que la finalidad de la retroalimentación es cerrar la brecha entre lo que se sabe en el momento actual y lo que debe aprenderse. Asimismo, estos autores expresan que es aquella información acerca de cómo se ha llevado a cabo un procedimiento, o del grado de comprensión de un proceso, que puede ser brindada por un compañero al ofrecer alternativas de solución, o por un libro que permite clarificar ideas, también puede ofrecerla un adulto (padre de familia u otro) que orienta y motiva, o bien, por el profesor que da a conocer pautas o pistas respecto de si se ha efectuado correctamente o no un procedimiento.

La idea de que la retroalimentación permite cerrar la brecha entre lo aprendido y lo enseñado es compartida por Wiggins (1998, en Ravela, Picaroni, Loureiro, 2017: 153), quien además agrega que "en lo posible no debería incluir valoraciones, sino más bien descripciones, [...] es altamente específica, directamente reveladora o altamente descriptiva de lo que realmente sucedió".

Es importante precisar la idea de Narcis (2008, en MacDonald, 2015) respecto de que la retroalimentación no solo da a conocer a los estudiantes información de sus errores y debilidades, sino que también les da sugerencias acerca de cómo mejorar su desempeño en situaciones de aprendizaje futuros. La idea anterior es apoyada por Wiliam (1999: 10) cuando afirma que la retroalimentación "no debería decirle a los estudiantes que trabajaran más duro, o que fueran más sistemáticos, sino debería contener pautas para la acción futura, de otro modo no sería formativa".

La retroalimentación puede ser proporcionada por diferentes personas o mediadores de aprendizaje: los compañeros, el docente, padres de familia, otros adultos, o bien diversos recursos como libros u otro material ya sea físico o digital.

De acuerdo con Hattie y Gan (2011: 258)

la información que proporciona la retroalimentación puede ayudar al estudiante a incrementar su esfuerzo, motivación o compromiso en la tarea, puede indicar que una respuesta es correcta o incorrecta, 
puede indicar que se necesita una mayor cantidad de información, puede señalar la dirección que el estudiante debe seguir para lograr el resultado que se espera, o indicar estrategias alternativas para entender una información en particular.

En las definiciones anteriores de este concepto se expresa la importancia de la especificidad de la información que debe proporcionarse, y se pone de manifiesto que lo que se exprese mediante la retroalimentación debe describir, en forma clara, el procedimiento a seguir por el aprendiz para pasar del estado actual de conocimiento hacia el esperado. Por tanto, no debe limitarse a dictaminar positiva o negativamente el proceso, o a asignar una cantidad o literal de valoración, sino que debe dar una descripción respecto de cómo transitar del conocimiento actual hacia el esperado.

Respecto a la idea anterior, Wiliam (2012) afirma que no es suficiente decirle al alumno si está bien o no, ya que eso no basta, en cambio, es necesario un intercambio entre el profesor y el estudiante que haga que este último se involucre en la tarea del aprendizaje y comprenda el significado de la retroalimentación brindada.

Shute (2008), tras una extensa revisión de la literatura, plantea que una retroalimentación efectiva está caracterizada por tres elementos: a) un motivo, es decir, que el estudiante la necesite, b) una oportunidad, ya que los alumnos la reciben en un tiempo adecuado para poder hacer uso de ella, y c) es un medio, el cual es posible que los educandos utilicen ya que está a su disposición si lo desean.

Asimismo, el autor antes citado provee una guía de nueve elementos para la mejora del aprendizaje a través de la retroalimentación: 1) debe estar centrada en la tarea y no en la persona, 2) es necesario que sea lo suficientemente elaborada y dé a conocer el qué, el cómo y el por qué, 3) se da a los estudiantes en pequeñas dosis de modo que sea manejable 4) es necesario que sea específica y clara, 5) debe ser tan simple como sea posible, 6) reduce la incertidumbre entre la actuación y la meta de aprendizaje, 7) es imparcial y objetiva, 8) promueve un aprendizaje orientado a metas, 9) se provee después de que los alumnos han intentado dar una solución correcta, favoreciendo la autorregulación.

Para continuar con la visión de la retroalimentación desde la perspectiva de los alumnos, Cizek (2010) afirma que el papel del estudiante es fundamental en el proceso de retroalimentación formativa ya que, más que cómo se brinde por parte del profesor, es necesario que sea recibida por los educandos, solo en la medida que la acepten y la pongan en juego, es posible lograr mejoras en su aprenidzaje (Hattie, Gan, 2011). Debido a lo anterior, uno de los objetivos principales del presente estudio es indagar qué representa para los alumnos el hecho de recibir retroalimentación altamente descriptiva, conocer en qué medida la usan y valorar la efectividad que tiene para su aprendizaje.

Además, una idea que da continuidad a las aportadas en los dos párrafos precedentes es que la retroalimentación que se le dé a los estudiantes debe fomentar que piensen y reflexio-

Diálo@os

sobre Educación año 12 | número 22 | enero-junio 2021 | ISSN 2007-2171 
nen, ya que si no se logra este cometido la retroalimentación no tiene un carácter formativo (Hattie, 2003b, en Shrum, 2016).

Por otro lado, Wimme y Butler (1994, en Hattie, Timperley, 2007: 82) afirman que "la retroalimentación es información con la cual un aprendiz puede confirmar, agregar [...] o reestructurar información en la memoria, ya sea información, conocimiento metacognitivo, creencias sobre sí mismo y las tareas, o tácticas y estrategias cognitivas".

Estos autores, a diferencia de las definiciones citadas, expresan que la retroalimentación puede tener la finalidad de incidir directamente en contenidos factuales, o bien puede estar dirigida a la mejora de las estrategias que emplean los estudiantes para resolver problemas o estar destinada a favorecer las actitudes y motivación de los educandos.

Algunos otros elementos que destaca Wiggins (1998, en Ravela, Picaroni, Loureiro, 2017: 154) respecto de una retroalimentación adecuada son:

Brinda al estudiante evidencias concretas sobre su trabajo, que le permiten confirmar o no la pertinencia del mismo. El alumno puede comparar lo que hizo con lo que se esperaba.

Ofrece ejemplos o modelos de tareas similares que satisfacen los criterios de calidad que debe alcanzar la misma, para que el propio estudiante saque sus conclusiones.

Este autor hace un añadido respecto de las características mencionadas respecto del concepto de retroalimentación: que pueden darse a conocer procedimientos terminados para que el estudiante compare su proceso con otros. Es decir, que a través del comparativo el estudiante pueda recabar la información que le permita cerrar la brecha entre el aprendizaje actual y el que se espera logre desarrollar.

Otro elemento a destacar de la retroalimentación es que es efectiva cuando el profesor brinda numerosas oportunidades a los alumnos para que la busquen y la reciban (Pollock, 2012), ya que esta puede obtenerse de distintos agentes, tales como: los compañeros, algún medio tecnológico, entre otros, como ya se ha apuntado, no solo de él.

Además, de acuerdo con Brookhart (2012), dos elementos para que la retroalimentación sea efectiva son, primero, que se brinde en un tiempo adecuado, lo que significa que el profesor brinda a sus estudiantes la posibilidad de, segundo, utilizarla en el momento. Es decir, cuando los estudiantes aún están pensando respecto de su trabajo, lo cual les permitiría poner en práctica la información recibida. Puesto que de nada sirve la retroalimentación si no se da a los alumnos la oportunidad de utilizarla en forma más o menos inmediata.

Otros autores coinciden con el planteamiento anterior y afirman que la retroalimentación debe ser: correctiva, brindarse en un tiempo adecuado, así como referir a criterios previamente acordados (Marzano, Pickering, Pollock, 2001), lo que traerá como resultado una mayor efectividad de esta estrategia para el aprendizaje. 
Lamentablemente, el problema en general es que, de acuerdo con Hattie y Timperley (2007), los estudiantes solo reciben unos pocos segundos por día de retroalimentación altamente descriptiva que pueda beneficiar su aprendizaje, cuestión que se quiere transformar a través de la propuesta que surge de este estudio.

En síntesis, algunos atributos fundamentales identificados por distintos referentes teóricos, respecto del concepto de retroalimentación son:

- Es la información que permite al estudiante conocer qué ha aprendido hasta el momento, qué le falta por aprender y qué necesita hacer a fin de llegar al aprendizaje que se espera logre consolidar. Por lo que algunos autores lo han denominado la brecha entre el nivel de desempeño actual y el esperado.

- Dicha información puede ser proporcionada por distintas personas o agentes de mediación (un compañero, el maestro, el libro de texto, un material audiovisual, entre otros).

- Puede incidir en un contenido factual, procedimental, actitudinal o influir en los procesos metacognitivos.

- Es importante que sea descriptiva y no se limite a señalar si lo realizado es correcto o incorrecto.

- Puede brindarse dando a conocer al estudiante productos terminados, para que compare su desempeño con procesos concluidos.

- Se da en un tiempo oportuno, cuando el estudiante está pensando en la tarea de aprendizaje, lo que le da la posibilidad de que utilice la información para mejorar su desempeño.

Resulta muy importante hacer una distinción entre la conceptualización de retroalimentación desde la que parte esta investigación (Aprendizaje y Enseñanza Visible, de Hattie, Timperley, 2007), y contrastarla con otras perspectivas. Puesto que, por ejemplo, de acuerdo con el objetivismo, dentro del cual se encuentra el conductismo, la retroalimentación es un reforzador en un modelo input-output, que funciona de manera automática, independientemente de las características de los sujetos.

Por el contrario, desde la perspectiva de esta investigación, la información brindada a los estudiantes para la mejora de su desempeño puede aceptarse, modificarse o rechazarse, de modo que lleve al alumno a la autorregulación. Asimismo, la retroalimentación, por sí misma, no tiene la capacidad para desarrollar una acción adicional del alumno (Hattie, Timperley, 2007).

Es decir, a pesar de que se considera a la retroalimentación como un medio importante para favorecer el aprendizaje, el solo hecho de brindarla o hacerlo a modo de reforzador (como un castigo o un premio), no traerá consigo una respuesta de forma automática, como es vista en el paradigma conductista, sino que debe brindarse en una situación de aprendizaje correctamente diseñada, además de propiciar que el estudiante la utilice. 


\section{El modelo Kieren-Behr}

Un modelo que permitió organizar el trabajo con las fracciones fue el iniciado por Kieren y complementado por Behr (1976, en Charalambous, Pitta-Pantazi, 2007), mismo que fue adaptado para la construcción de las actividades de la aplicación Retromath que brindó retroalimentación a los estudiantes en el presente estudio.

Este modelo considera como una de las principales causas del pobre aprendizaje de las fracciones la existencia de cinco subconstructos (Razón, operador, cociente, medida y partetodo) asociados al concepto de fracción, que lo complejizan y no se toman en cuenta durante el proceso de enseñanza por parte de los profesores.

\section{La aplicación Retromath}

Como se ha puesto de manifiesto en apartados anteriores, se ha diseñado y desarrollado la aplicación Retromath en función de las características requeridas para que proporcione retroalimentación de acuerdo con el modelo propuesto por la teoría del aprendizaje visible de Hattie y Timperley (2007); asimismo, tiene como principal propósito la resolución de problemas que involucren números fraccionarios de los estudiantes de quinto de primaria.

A fin de contar con esta herramienta fundamental para el estudio, se estableció una relación de colaboración con una estudiante de la Maestría en Tecnologías de Información, del Centro Universitario de Ciencias Económico Administrativas (CUCEA), para que ella trabajara en la parte tecnológica.

En el mes de octubre de 2019 se entregó la propuesta didáctica completa que incluía 131 reactivos, textos de retroalimentación, las imágenes que contendrían los elementos anteriores, la manera en que estarían organizados los problemas de acuerdo con los subconstructos propuestos por Kieren y Behr (1976 en Charalambous, Pitta-Pantazi, 2007), así como con cuatro niveles de dificultad.

En los meses subsiguientes se tuvieron reuniones con la maestrante, su director de tesis, la directora de la presente investigación, así como otros docentes del CUCEA que revisaron la propuesta y dieron sugerencias de mejora.

En lo referente a la parte tecnológica, se establecieron fechas para la realización de pruebas de la aplicación, no obstante, estas fueron postergadas en reiteradas ocasiones. Fue hasta el mes de marzo de 2020 cuando se pudo observar la funcionabilidad de la misma, pero debido a la contingencia por la pandemia mundial provocada por el COVID-19, no se pudieron realizar de forma presencial en la escuela primaria.

Durante la segunda mitad de abril del mismo año, se decidió modificar la manera de realizar el proyecto e interactuar con algunos estudiantes de forma remota, debido a que todos los alumnos estaban tomando sus clases a distancia. En ese contexto, se realizaron pruebas con dos estudiantes y una vez que se verificó que la aplicación contaba con los requerimientos 
técnicos y el trabajo con ella hacía posible dar respuesta a las preguntas de investigación, se diseñó el presente estudio que sería llevado a cabo durante las tres últimas semanas de mayo y la primera de junio de 2020.

Para el diseño de dicha aplicación, en lo que respecta al contenido de las fracciones, se tomó en cuenta el modelo de Kieren y Behr (1976, en Charalambous, Pitta-Pantazi, 2007) así como el Programa de Estudios vigente (SEP, 2011), adoptando la decisión de trabajar con tres subconstructos propuestos por el modelo antes mencionado: parte de un todo, medida y reparto, ya que el programa del grado escolar de los estudiantes sugiere abordarlos.

\section{Organización de la aplicación}

La aplicación cuenta con 131 problemas, planteados mediante reactivos de opción múltiple. Están distribuidos en cuatro niveles de dificultad, y organizados en tres secciones que corresponden a tres de los cinco subconstructos propuestos por el modelo de Kieren y Behr.

Contiene un elemento que proporciona retroalimentación de acuerdo con el modelo de Hattie y Timperley (2007): un avatar que debe ser seleccionado por cada estudiante al comienzo del juego, el cual retroalimenta de forma automática si la manera de contestar fue correcta o no, y propone al estudiante recibir información de nivel de proceso, si es que lo desea.

\section{Metodología}

En el estudio se utilizó una perspectiva metodológica cualitativa que, de acuerdo con Creswell (2012), ayuda al investigador a desarrollar la comprensión de un fenómeno. Dentro de ella se utilizó un estudio cualitativo de caso.

Quienes emplean la metodología cualitativa buscan explorar un problema a través de la recolección de datos mediante la observación y/o las entrevistas, para construir conocimiento y entender un fenómeno (Creswell, 2012).

En función de lo anterior, se obtuvieron datos cualitativos mediante la observación participante, además de entrevistas semiestructuradas, que fueron videograbadas, transcritas y analizadas mediante un doble ciclo de análisis (Saldaña, 2009).

\section{Métodología cualitativa}

De acuerdo con Creswell (2012), en la perspectiva cualitativa la intención principal no es obtener generalizaciones, sino desarrollar una exploración en profundidad del fenómeno estudiado.

Asimismo, Flick (2007) expone algunos de sus rasgos principales: partir de las perspectivas de los participantes y su diversidad, la capacidad que debe tener el investigador y la investigación para generar reflexión, además de que dentro de ella existe una gran variedad de enfoques y métodos.

En relación con el último punto, Rathje (2018) distingue entre métodos que buscan construir teoría, como la teoría fundamentada, o aquellos que pretenden entender las interacciones 
de los participantes al interior de una cultura como la etnografía. Asimismo, están aquellos que ponen especial atención en las perspectivas de los sujetos, sus creencias y su perspectiva individual, como son los estudios fenomenológicos.

En el presente trabajo no se optó por ninguno de los métodos citados; debido a que su intención es comprender la manera en que la retroalimentación favorece el aprendizaje de los estudiantes, es decir, que su mirada no se pone directamente en los sujetos y sus creencias - ante lo cual sería conveniente utilizar la fenomenología - sino en comprender cómo utilizan la retroalimentación y la manera en que favorece la construcción de nuevos aprendizajes, se optó por emplear un estudio de caso cualitativo.

De acuerdo con Yin (2018: 45), un estudio de caso es un método empírico que investiga "un fenómeno contemporáneo (el caso) en profundidad y dentro de su contexto real. [...] se podría querer hacer un estudio de caso porque se quiere comprender un caso del mundo real y asumir que tal comprensión es posible para envolver importantes condiciones contextuales".

\section{Desarrollo}

En la presente investigación se estudiaron cuatro estudiantes, quienes interactuaron con una aplicación y con el investigador de manera remota a través de videoconferencia, que en el contexto de contingencia por la pandemia representó su contexto escolar, para comprender la manera en que utilizaron la retroalimentación para aprender.

Por las razones anteriores y de acuerdo con los aportes de Stake (1999), se llevó a cabo un estudio instrumental de caso ya que analizar la manera de actuar de los alumnos permitió comprender el rol que jugaba la retroalimentación para la mejora del aprendizaje.

Debido a que cuatro estudiantes participaron en la investigación, el uso de múltiples casos hizo posible identificar hallazgos comunes, así como diferencias en todos ellos y utilizar un análisis cruzado de casos (Creswell, 2012), lo cual posibilitó enriquecer los hallazgos.

Para la recolección de los datos se siguieron los pasos para la investigación cualitativa propuestos por Creswell (2012), mismos que dan lugar a los apartados que a continuación se exponen.

\section{Selección de los participantes}

Como ya se mencionó, durante la planeación de la investigación se había seleccionado una escuela del municipio de Zapopan, Jalisco, y dos de sus grupos de quinto grado para realizar el estudio. No obstante, dada la situación de contingencia y la necesidad de continuar con el trabajo de campo, se modificó la manera de llevar a cabo el trabajo, así como los participantes.

Para su selección se solicitó a algunas autoridades educativas del municipio de Yahualica, Jalisco, un supervisor de nivel de primaria, la directora de una escuela particular, y dos maestros (uno de una escuela pública y otro de escuela privada), que hicieran la invitación a algunos estudiantes para participar en el trabajo. Quienes desearan ser parte de él necesitarían ser alumnos de quinto grado, contar con una computadora y acceso a Internet. 
El caso comprendió (Stake, 1999) cuatro estudiantes que aceptaron participar. La primera sesión consistió en dialogar con ellos, así como realizar un examen diagnóstico del contenido a abordar. Durante el estudio fue posible darse cuenta que la muestra contaba con una máxima variación (Creswell, 2012): dos hombres y dos mujeres, dos de los estudiantes tenían un dominio importante del contenido, mientras que los otros manifestaron dificultades para resolver problemas fraccionarios; un par de alumnos vivían dentro de la ciudad, y los otros en colonias fuera de la misma. Asimismo, el carácter de un y una estudiante era reservado e introvertido, mientras que los dos restantes eran extrovertidos.

\section{Permisos de acceso}

Como se mencionó en el apartado anterior, el hecho de solicitar el apoyo de distintas autoridades educativas facilitó que los padres accedieran a que sus hijos participaran en el estudio. Vía telefónica se les explicó en qué consistiría la actividad, los requisitos, así como algunos beneficios de participar en ella. Los padres estuvieron de acuerdo y se les pidió que firmaran el consentimiento informado, en el que autorizaban la participación de sus hijos durante tres semanas en el trabajo.

\section{Ética de la investigación}

Cuando se recolectó la firma de consentimiento informado, se explicó a los padres que ningún dato personal sería dado a conocer en el informe de la investigación; asimismo, se les dijo que se utilizarían pseudónimos para guardar el anonimato.

Durante las actividades de la investigación: utilización de la aplicación por parte de los estudiantes y entrevistas, se permitió que los padres observaran y escucharan el desarrollo de las actividades. A los que decidieron observar la actividad, con sutileza se les comentó que permitieran que los alumnos resolvieran los problemas y tomaran decisiones sobre el uso de la aplicación de manera independiente.

\section{Instrumentos de recolección de datos}

Durante el análisis de caso fueron necesarios dos instrumentos para la recolección de datos: la observación participante y la entrevista. Asimismo, dadas las características del estudio: alumnos interactuando con una aplicación mientras eran observados por el investigador, se vio necesario realizar una entrevista al final de cada sesión para recoger sus impresiones; todo el proceso fue videograbado y después transcrito para su análisis.

\section{Observación participante}

De acuerdo con Creswell (2012), la observación es el proceso de obtener información de primera mano de personas o lugares, y tiene la ventaja de que se puede conseguir una gran cantidad de datos aun si los participantes tienen dificultades para expresarse.

Diálo@os año 12 | número 22 | enero-junio 2021 | ISSN 2007-2171 
Existen diferentes roles que puede adoptar el investigador de acuerdo con el grado de interacción que tenga con las personas que participan en el estudio. En este caso, se adoptó un rol de observador participante ya que, además de observar y recolectar notas de lo percibido, se tomó parte en las actividades (Creswell, 2012).

\section{La entrevista}

A fin de contar con otra técnica que aportara información cualitativa, se planeó realizar entrevistas con los alumnos participantes; para ello se tomaron en cuenta los criterios que expone Kvale (2011).

Las entrevistas con los estudiantes fueron semiestructuradas. Estas son definidas como "entrevistas con el propósito de obtener descripciones del mundo de la vida del entrevistado respecto a la interpretación del significado de los fenómenos descritos" (Kvale, 2011: 30). Se pretendió obtener la descripción del estudiante respecto de su experiencia con la utilización de la aplicación y de cómo vivenció los distintos tipos de retroalimentación que le fueron proporcionados, así como conocer de qué manera favorecieron su aprendizaje, saber si fue una situación nueva o si ya había trabajado con una plataforma similar, si fue de su agrado, si le gustaría continuar aprendiendo otros contenidos de esa manera, etcétera.

\section{La videograbación}

Como se mencionó en el párrafo introductorio, uno de los instrumentos de recogida de datos que se utilizó durante la investigación fue la videograbación.

Debido a que uno de los propósitos del estudio es comprender la manera en que la retroalimentación favorece el aprendizaje de los estudiantes en situaciones de aprendizaje mediadas por la computadora, fue fundamental observar los comportamientos e interacciones de los alumnos, así como la manera en que se relacionaron con los elementos tecnológicos.

La videograbación se utilizó con una perspectiva interpretativa, siguiendo los planteamientos de Knoblauch y Tuma (2011), y permitió recuperar de forma fidedigna las interacciones de los alumnos con la aplicación, así como las de ellos con el investigador, haciendo posible recuperar los diálogos que se tuvieron a lo largo del estudio, así como los que se tuvieron durante la entrevista.

Las potencialidades identificadas en la videograbación se deben a su capacidad para revisar permanentemente la situación tal y como ocurrió, debido a la posibilidad de repetir, dar zoom en una situación específica, observar la situación en cámara lenta, etcétera (Knoblauch, Tuma, 2011).

Como el lector puede darse cuenta, el tipo de videograbación que se empleó posee características peculiares, ya que no solo era necesario conocer la manera en que se comportan los estudiantes, sino también analizar qué acciones realizaron con la computadora. 
Por ello se recurrió a la técnica denominada observación remota, la cual, de acuerdo con Donoso Vegas y González Figueroa (2013: 84), sirve para "observar el comportamiento en pantalla de un usuario de la red. Esta se basa en la observación en tiempo real de la actividad de un grupo de computadores utilizando las herramientas que en general provee un software de administración de red". Como lo apuntan los autores tiene la ventaja de ser un instrumento de recogida de información no invasivo, y permitió que los usuarios interactuaran de manera natural con la computadora.

A fin de lograr lo anterior, se empleó el programa Camtasia, que ha sido utilizado de manera semejante en otros estudios (Kriscautzky, Ferreiro, 2017; Donoso-Vegas, González-Figueroa, 2013); es conceptualizado por Donoso Vegas y González Figueroa (2013) como "un software open source [...] que permite la grabación de videos en formato AVI y SWF y la captura de secciones completas de la pantalla [...] Cuenta con la posibilidad de ocultar el uso del software mientras este se encuentra grabando".

Se asume que estas características fueron ideales para el trabajo ya que hicieron posible conocer lo que el estudiante realizaba en la computadora al mismo tiempo que se observaban sus gestos e interacciones con otros, así como las expresiones que surgían del contacto con los recursos de la aplicación.

De acuerdo con la propuesta de videograbación sugerida por Knoblauch y Tuma (2011), el investigador determina la situación focalizada. En el presente trabajo, se centró en observar la interacción de los estudiantes con la aplicación y con el investigador.

\section{Trabajo con la aplicación}

Durante cada una de las sesiones se observó a los alumnos en forma individual a través de la cámara web y se les solicitó que compartieran su pantalla de manera que era posible observar su rostro, así como lo que estaban trabajando en la aplicación.

Al inicio de la sesión, el investigador les apoyaba en las cuestiones técnicas: diciéndoles de qué manera podrían entrar a la aplicación. Una vez ahí, contestaban los ejercicios propuestos, mismos que se presentaron de la siguiente manera: 
Figura 1. Problema número 126, del tema: Fracción como medida, nivel 4

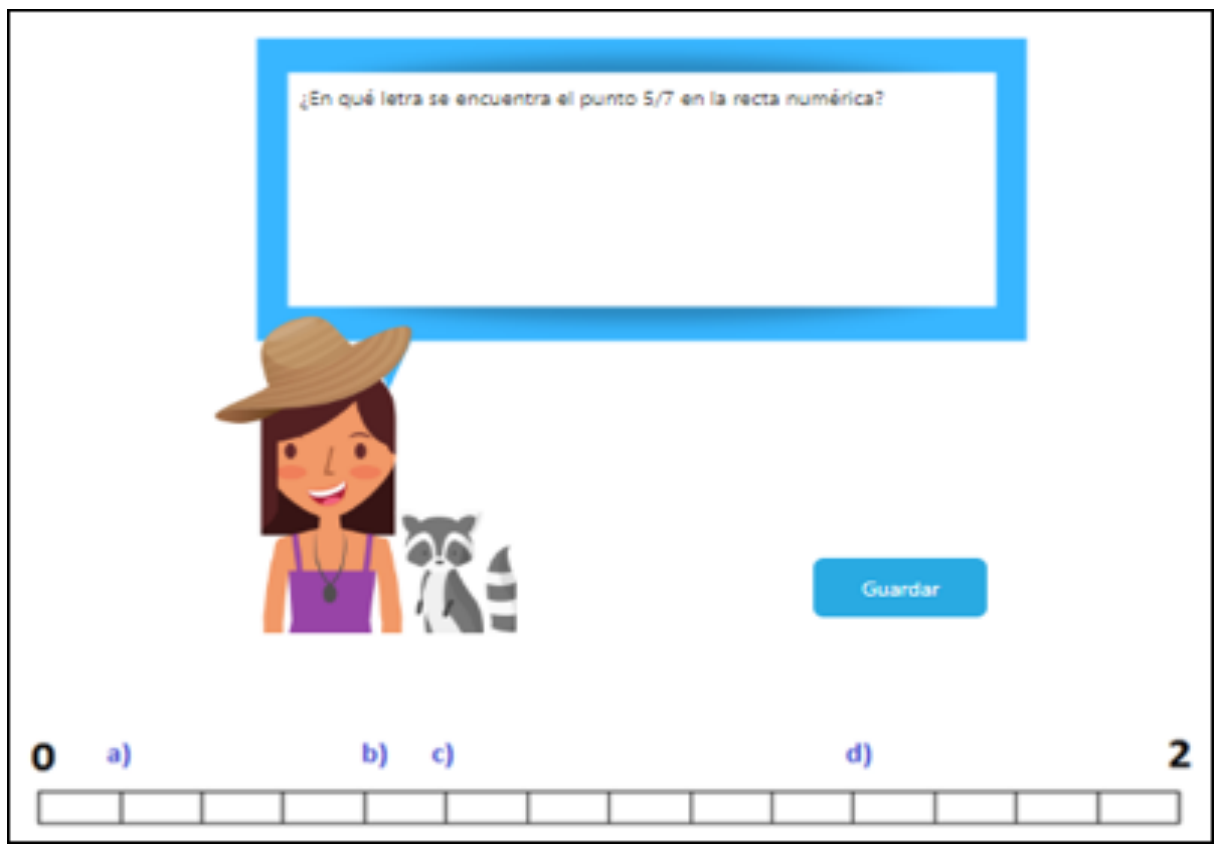

En el ejemplo anterior se muestra el problema número 126, correspondiente al subconstructo fracción como medida (Kieren, Behr, 1976 en Charalambous, Pitta-Pantazi, 2007), clasificado como un ejercicio de nivel 4 (en el nivel 1 se encuentran los problemas más sencillos, y conforme se avanza en la resolución de los mismos se complejizan hasta llegar al nivel 4, el más complejo con que cuenta la aplicación), cuyo propósito es identificar en una recta numérica una fracción dada, de la que solo se tienen los referentes de inicio (el punto 0) y fin de la recta (el punto 2), sin mencionar a cuánto equivalen cada una de las subdivisiones, no incluyendo pistas respecto de esta información, solo el número de marcas en que se divide la recta.

Una vez contestado el ejercicio, y recibida la retroalimentación de la tarea que da a conocer si el ejercicio fue contestado correctamente o no, se da la posibilidad al alumno de acceder a la retroalimentación de proceso, tanto si respondió bien como si lo hizo mal. En caso de que acepte, se le brindan los siguientes mensajes: 
Figura 2. Mensaje de retroalimentación número 1 del problema 126

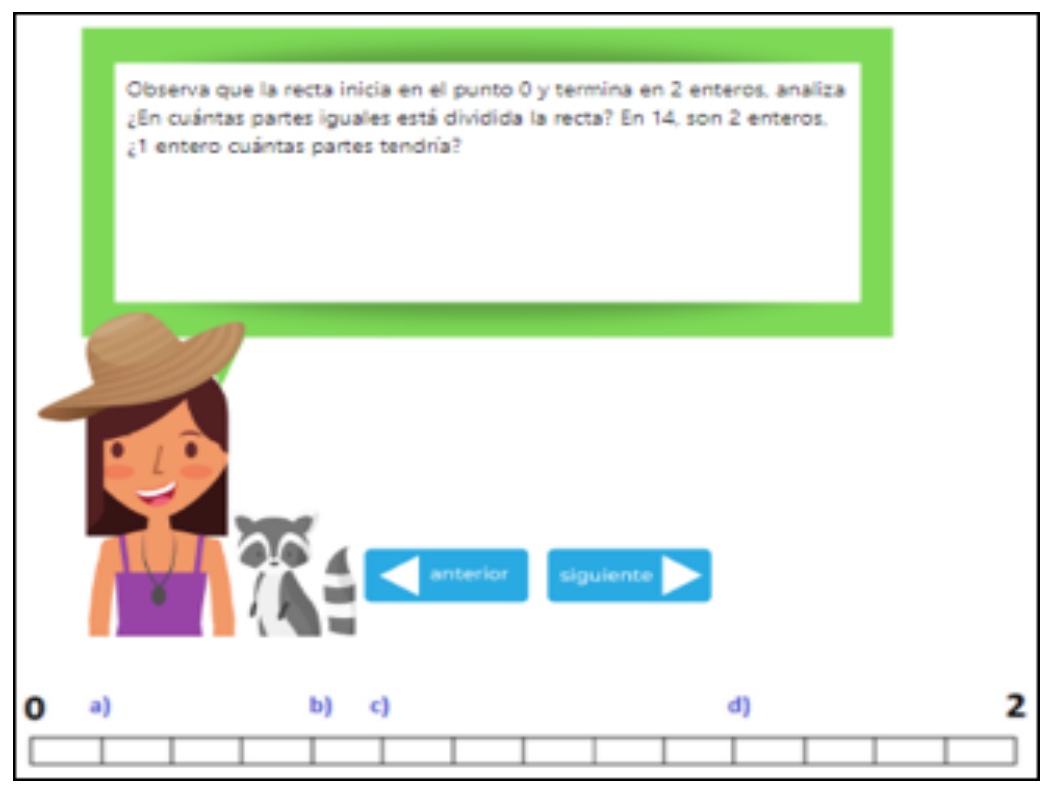

Mediante este mensaje se busca centrar a los estudiantes en la importancia de identificar las cantidades del punto inicial y el punto final de la recta numérica. Enseguida, analizar en cuántas partes está dividida la misma. En este caso se pone énfasis en que inicia en un entero y termina en el punto 2 enteros. Además, tiene la finalidad de corroborar el número total de veces en que está dividida la recta, así como aclarar que esas divisiones son para dos enteros, y que habrá que considerar en cuántas partes está dividido uno de ellos. Por esta razón se lanza la siguiente pregunta al estudiante: ¿Un entero cuántas partes tendrá?

Después, se les proporciona el siguiente mensaje: 
Figura 3. Mensaje de retroalimentación número 2 del problema 126

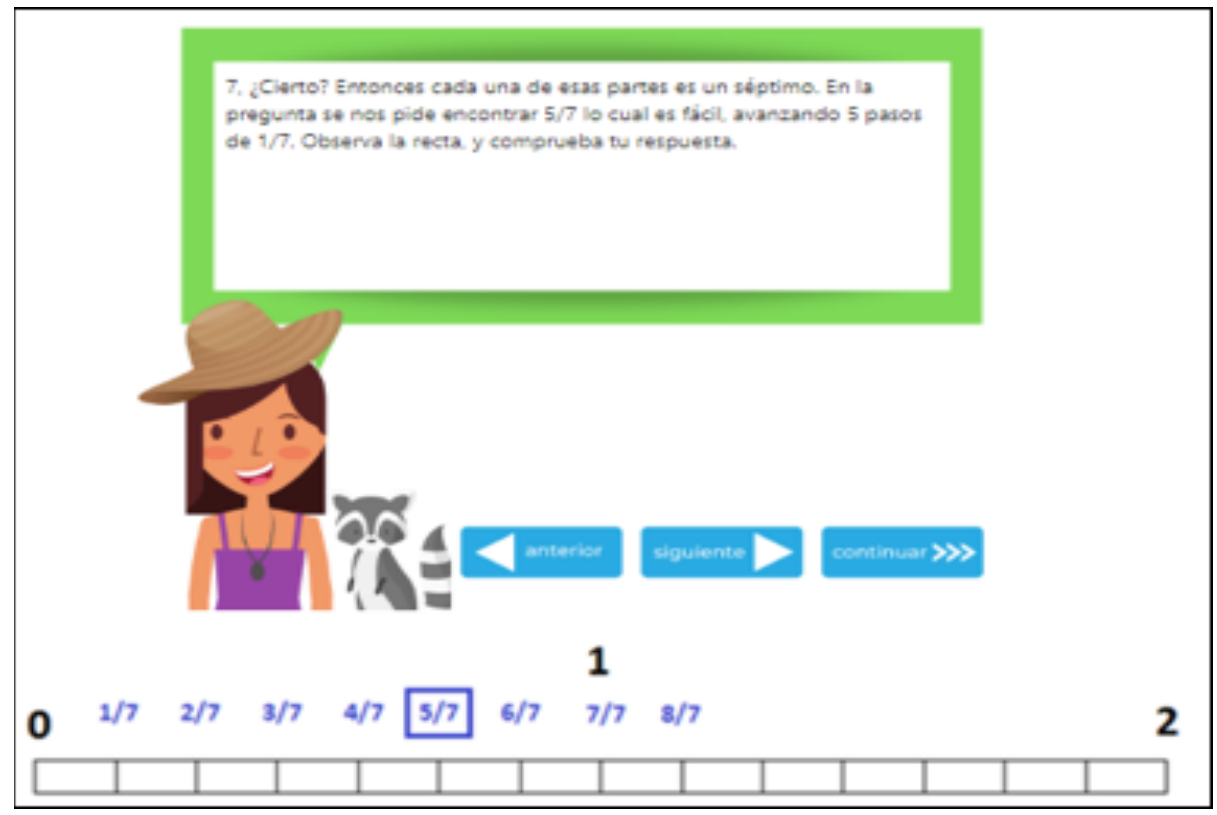

La segunda parte de la retroalimentación se da mediante un diagrama y el texto que brinda respuesta a la pregunta hecha en el mensaje anterior, asimismo, se da a conocer que, dado que cada entero está dividido en 7 partes iguales, cada una corresponde a 1/7, y solo hay que contar 5 segmentos, partiendo de 0 para encontrar el punto 5/7, que se solicitó en el enunciado del problema.

De esta manera, se sugiere al estudiante un proceso a seguir mediante la retroalimentación. Para resolver problemas de identificación de fracciones en una recta numérica, que contiene como referencia el punto inicial y final, así como un determinado número de subdivisiones, el alumno podría seguir un proceso como el que se describe a continuación:

- Observar el punto inicial y final de la recta numérica.

- Identificar el número de subdivisiones de la misma.

- Analizar cuántas subdivisiones corresponden a un entero.

- Determinar el valor de las subdivisiones.

- Identificar el punto que se pide en el enunciado problema.

El resto de los ejercicios cuenta con retroalimentaciones brindadas de manera semejante, variando los conceptos de acuerdo al tipo de problema que se está resolviendo.

Es importante destacar que, para validar el efecto de la retroalimentación, cuando un alumno se equivocaba en un problema, se le asignaba otro del mismo nivel y subconstructo, para 
que tuviera la oportunidad de utilizar la retroalimentación proporcionada por la aplicación en caso de haberla aceptado.

Asimismo, a los estudiantes que durante el trabajo tenían dificultades con el contenido, el investigador les hizo preguntas y brindó sugerencias, como las que se observan en la siguiente viñeta: (Mo, alude al escritor del presente trabajo quien coordinó las actividades, en tanto que Ao, se refiere al alumno que fue partícipe del estudio, el nombre propio utilizado en el recorte es un pseudónimo).

Mo: A ver, Ismael, espérame.

Ao: (Se equivoca en un problema que pide seleccionar qué es más grande: $2 / 40$ 1/8. Pone cara de frustración y pide retroalimentación).

Mo: Tranquilo, no pasa nada, vuélvelos a ver. Dale para adelante, dale (Le pide que avance en los mensajes de retroalimentación proporcionados por la aplicación). Ahí, espérame. Mira, ¿te acuerdas?, ya habías dicho: ¿Cuáles son más grandes? ¿Los cuartos o los octavos?

Ao: ¿Cuáles son más grandes? los cuartos.

Mo: Los cuartos, entonces, fíjate, si un solo cuarto es más grande que un octavo, jimagínate $2 / 4$ !, fíjate hasta dónde llega 2/4 (Le indica que observe la imagen de la pantalla que compara gráficamente 2/4 con 1/8) ¿Cuántos octavos necesitas para tener dos cuartos?

Ao: ¿Cuántos octavos necesito para tener dos cuartos? ¿4 octavos?

Mo: Cuatro octavos se necesitan para tener dos cuartos, ¿verdad? ¿Sí te fijaste en eso?

Ao: Sí.

(Sesión 3_Ismael, Pos. 135-142).

En el recorte anterior, tomado de la tercera sesión de trabajo con un estudiante que tenía dificultades para resolver problemas fraccionarios, se observa que además de la retroalimentación que proporcionaba la aplicación Retromath fue necesaria la intervención del investigador para apoyar conceptualmente al alumno. Este tipo de intervenciones solo ocurrieron con dos de los casos, ya que el resto pudo trabajar de manera autónoma y, en caso de error, les bastaba recibir la retroalimentación de la aplicación para continuar avanzando.

\section{Análisis de la información}

A fin de realizar un análisis exhaustivo de la información recabada mediante la observación, las entrevistas y los datos obtenidos a través de la videograbación, se llevó a cabo el proceso de transcripción de los datos para posteriormente analizarlos. Para ello se tomaron en cuenta los planteamientos de Saldaña (2009) y se utilizó un doble ciclo de codificación, de lo cual se da cuenta en este apartado. Como resultado del proceso antes señalado surgen cuatro temas: 1 ) Percepciones de los estudiantes respecto de la retroalimentación, 2) Maneras en que los estu-

Diálo@os sobre Educación año 12 | número 22 | enero-junio 2021 | ISSN 2007-2171 
diantes utilizan la retroalimentación, 3) Elementos que complejizan el proceso de retroalimentación, y 4) Relación entre retroalimentación y aprendizaje. Ellos hicieron posible comprender de mejor manera el fenómeno estudiado, así como redactar algunos hallazgos y conclusiones.

\section{Doble ciclo de codificación}

El primer paso, previo al análisis, consistió en transcribir las videograbaciones de cada una de las sesiones de trabajo que tuvieron los estudiantes, en las cuales interactuaron con la aplicación y con el investigador, además de que fueron entrevistados al final de todas ellas.

Una vez transcrita la información con ayuda del Programa MaxQDA (Rädiker, Kuckartz, 2020) se inició el proceso de codificación de acuerdo con los planteamientos de Saldaña (2009).

Para el autor antes citado, un código es "una palabra o frase corta que simbólicamente se asigna a una porción de lenguaje o dato visual destacado, y capturador de esencia" (2009: 3), estos se encuentran buscando patrones de similitud, diferencia, frecuencia, correspondencia, causa, etcétera. Asimismo, el acto de codificar consiste, de acuerdo a sus planteamientos, en la acción de organizar y agrupar los códigos en unidades o familias con el mismo significado.

Existen distintos métodos para realizar este proceso y se les distingue en cuanto a su complejidad en métodos de primer y segundo ciclo.

El método de primer ciclo utilizado en esta investigación fue el de codificación descriptiva, el cual "Resume en una palabra o frase corta, casi siempre con un sustantivo, el tema básico de un pasaje del dato cualitativo. [...] se les pueden añadir más sub-códigos cuando sea necesario. [...] La codificación descriptiva lleva a un inventario de categorías, a una cuenta tabular, o índice de datos de contenidos (Saldaña, 2009: 72).

Para realizar el proceso se identificaron segmentos de las transcripciones con palabras o frases que permitieran reconocer los hechos o palabras a los que se referían con facilidad. Debido a que existieron muchas regularidades durante las sesiones, tales como: iniciar con el saludo y cuestiones de rapport, abrir la aplicación, comenzar el juego, etc., fue relativamente sencillo encontrar similitudes e iniciar la codificación descriptiva.

En la segunda etapa se empleó la codificación por patrones (Saldaña, 2009), que busca la unión de una gran cantidad de datos significativos, hasta llegar a la construcción de metacódigos o temas; para ello se redacta un enunciado que describa la red de interrelaciones encontradas o un constructo teórico.

Debido a que la idea anterior se encuentra muy relacionada con las ideas de Stake (1999), quien propone que para los estudios de caso es importante la construcción de temas que favorezcan el análisis, se obtuvieron cuatro temas mediante los cuales fue posible dar cuenta de lo acontecido con los estudiantes que participaron en el estudio y establecer hallazgos, limitaciones y conclusiones. 


\section{Resultados}

Los hallazgos más importantes de la investigación tienen que ver con la manera en que los estudiantes conceptualizaron y utilizaron la retroalimentación, sobre todo en su nivel de proceso y en el momento denominado feedforward (Hattie, Timperley, 2007), con las dificultades que encontraron los estudiantes para su utilización, así como con la importancia de la creación de ambientes de aprendizaje en que se reciba y utilize la retroalimentación para el aprendizaje.

\section{Conceptualización y uso de la retroalimentación}

De acuerdo con Hattie y Gan (2011), más importante que la manera en que el profesor da la retroalimentación es cómo los estudiantes la reciben y la utilizan para mejorar su aprendizaje.

El presente trabajo dio cuenta que para los estudiantes es importante recibir retroalimentación, es el elemento que más les agradó del juego en el que participaron, según lo expresaron en las entrevistas. De acuerdo con sus palabras, querían saber la manera en que podrían actuar para resolver los problemas.

Asimismo, en la mayoría de los casos no les bastaba con saber que su resultado había sido correcto o incorrecto (retroalimentación en su nivel de tarea), sino que les interesaba mejorar sus procedimientos (retroalimentación en el nivel de proceso), mediante las ayudas que les brindaba su avatar.

Los estudiantes dieron a la retroalimentación el calificativo de: ayudas, ejemplos, explicaciones y procedimientos, que les facilitaban la resolución de los problemas, y sin los cuales sería difícil resolverlos.

Para ellos fue un elemento fundamental recibir retroalimentación de proceso, sobre todo cuando cometían errores, ya que solicitaron este apoyo en $90 \%$ de las ocasiones en que se equivocaron al intentar resolver un problema. Y no solo eso, sino que a lo largo del estudio fue posible observar cómo los educandos se apropiaban de los procedimientos de solución propuestos por su avatar, y los empleaban de forma paulatina para dar solución a las situaciones que se les presentaban.

En la mayoría de los casos se pudo observar la eficacia de la retroalimentación cuando no hubo reiteración de los mismos errores durante las distintas sesiones en que participaron. Solo en uno de los estudiantes esta situación no se presentó, debido a que su capacidad para resolver problemas fraccionarios era muy limitada, no obstante, pudo observarse una mejora en su conceptualización de las fracciones al resolver situaciones de forma autónoma durante las últimas sesiones.

\section{Dificultades para la utilización de la retroalimentación}

Como se mencionó durante los primeros apartados, la resolución de problemas fraccionarios es un contenido complejo para los estudiantes (Cortina, Zúñiga, Visnovska, 2013; González-Retana,

Diálo@os sobre Educación año 12 | número 22 | enero-junio 2021 | ISSN 2007-2171 
Eudave-Muñoz, 2018), esto se vio demostrado, sobre todo, en dos de los casos en que los estudiantes presentaron dificultades para la resolución de algunas problemáticas.

Para favorecer el aprendizaje de los estudiantes, en los últimos casos mencionados, además de la retroalimentación que se daba a través de la aplicación fue necesaria la intervención del profesor, quien mediante preguntas y sugerencias, ayudó a los alumnos en dos sentidos: en la comprensión de los números fraccionarios, y en la utilización de la retroalimentación al proponerles su empleo para la evolución de sus procedimientos de solución.

Algunas dificultades observadas en los estudiantes, principalmente en uno de los casos, fueron: deficiencias para la lectura: tanto de los textos de retroalimentación como de los números fraccionarios; apresuramiento por resolver los problemas sin analizarlos en profundidad; interrupciones de parte de las personas que los rodeaban mientras participaban en la actividad; deficiencias de redacción y otros elementos detectados en la aplicación durante el estudio.

En lo que respecta a las deficiencias en la lectura de los estudiantes, la aplicación tiene la capacidad de soportar archivos de audio, de modo que tanto las preguntas como los mensajes de retroalimentación pudieran contar con la versión escrita y la de audio, para que la comprensión lectora no fuera un impedimento para que los estudiantes pudieran mejorar en la resolución de problemas fraccionarios.

Asimismo, se ha gestionado la mejora de las fallas en los elementos de redacción de los problemas y enunciados de retroalimentación detectados en el estudio, tales como: repetición de respuestas en problemas u omisión de palabras, que, aunque leves, pudieron representar dificultades a los estudiantes. De este modo, se buscará que para futuros estudios la aplicación presente las mejoras señaladas.

En lo que respecta a las dificultades conceptuales presentadas por los alumnos, se ha concluido que la retroalimentación presentada por la aplicación es una ayuda importante para los educandos, pero debe ser potenciada por el acompañamiento y apoyo del profesor ya que algunos estudiantes requirieron de explicaciones puntuales para su mejora.

\section{Ambientes de aprendizaje propicios para la retroalimentación}

Uno de los elementos considerados de suma importancia por algunos autores (Temple-Adger, 2001; Hattie, Gan, 2011) para la aceptación y uso de la retroalimentación, son los ambientes de aprendizaje en los cuales el error sea visto con naturalidad y en los que incluso el profesor puede tener fallos, ya que estos son un paso natural hacia el aprendizaje.

Respecto a este elemento, pudieron observarse distintos comportamientos en los alumnos:

Tres de los educandos mostraron que eran capaces de aceptar el error y aprender de él; si bien se mostraban molestos cuando estos ocurrían, tenían la capacidad para aceptarlos, solicitar ayuda y continuar. Incluso en algunas ocasiones, cuando acertaban las preguntas pero habían 
tenido dudas en el procedimiento de solución, pedían ayuda ya que deseaban saber cómo se resolvían para, enseguida, ser capaces por sí mismos de resolver ese tipo de situaciones.

En contraste, uno de los estudiantes, al sumar una gran cantidad de errores durante las primeras sesiones, manifestó frustración, y en las sesiones subsiguientes ponía énfasis en obtener respuestas correctas y pasar de nivel, pero no tanto en comprender los procedimientos de solución. Asimismo, una de las cuestiones que se identificaron en este alumno durante una de las entrevistas, fue que asociaba los errores con regaños.

Este último elemento no hace posible la creación de un ambiente de aprendizaje que favorezca la retroalimentación, sino que privilegia que los estudiantes eviten los errores y se sientan mal ante ellos. Esta es una situación que se pretende transformar con los principios que sigue la aplicación, la cual favorece que los estudiantes busquen aprender y mejoren sus procedimientos, en un ambiente en el que no haya castigos ni recompensas.

\section{Resumen}

Los hallazgos encontrados en este estudio fueron que los estudiantes buscan la retroalimentación, sobre todo aquella en la que pueden identificar los procedimientos de mejora y la utilizan activamente para dar solución a las problemáticas que se les presentan.

Dada la complejidad del contenido abordado en el estudio, el solo hecho de brindar retroalimentación mediante la aplicación no fue suficiente para uno de los estudiantes y se requirió de la ayuda del docente para poder mejorar en su proceso de aprendizaje, lo cual será un hecho que probablemente suceda con los estudiantes que presentan dificultades en cualquier contenido.

Un elemento fundamental para que la retroalimentación sea recibida y utilizada en forma pertinente es la creación de ambientes de aprendizaje en los que el error sea aceptado y visto como una oportunidad, de modo que los estudiantes busquen la retroalimentación para la mejora de sus aprendizajes y no solo para obtener recompensas externas o evitar castigos. Como afirman Black y William (1998a, en Rathje, 2018: 8) "no es posible introducir la evaluación formativa sin un cambio radical en la pedagogía de los salones de clase". Lo cual implica una transformación en la manera de conceptualizar la evaluación y la retroalimentación de parte de todos los miembros de las comunidades educativas.

\section{Referencias}

Almendral, C. (2014). Formative Assessment through Written Feedback: Examining Elementary School Teachers' Written Feedback Beliefs and Practices, and the Effect of Models on Written

Diólopos 
Feedback. Tesis doctoral. Nueva York: Universidad de la Ciudad de Nueva York. https://academicworks.cuny.edu/cgi/viewcontent.cgi?article=1158\&context $=$ gc etds

Barrón Estrada, M.; R. Zatarain-Cabada; Y. Hernández-Pérez (2014). Tutor Inteligente con reconocimiento y manejo de emociones para Matemáticas. REDIE: Revista Electrónica De Investigación Educativa. http://redie.uabc.mx/index.php/redie/article/view/954/981

Block, D.; I. Fuenlabrada; H. Balbuena; A. Carvajal (1992). Juega y aprende matemáticas. Actividades para divertirse y trabajar en el aula. México: SEP.

Brookhart, S. M. (2012). Preventing Feedback Fizzle. Educational Leadership, 24-29.

Brooks, C.; A. Carroll; R. Gillies; J. Hattie (2019). A Matrix of Feedback for Learninng. Australian Journal of Teacher Education, 14-32.

Butler, R. (1988). Enhancing and Undermining Intinsic Motivation: The Effects of Task-involving and Ego-involving Evaluation on Interest and Performance. British Journal of Educational Psychology, 1-14.

Carless, D. (2006). Differing Perceptions in the Feedback Process. Studies in Higher Education, 219-233.

Charalambous, C. Y.; D. Pitta-Pantazi (2007). Drawing a Theoretical Model to Study Students' Understanding of Fractions. Educational Studies in Mathematics, 64(3), 293-316. http://www. jstor.org/stable/27822662

Cizek, G. (2010). An Introduction to Formative Assessment: History, Characteristics, and Challenges. En Andrade, H.; G. Cizek (eds.). Handbook of Formative Assessment. Nueva York: Routledge, 3-17.

Cortina, J. L.; C. Zúñiga; J. Visnovska (2013). La equipartición como obstáculo didáctico en la enseñanza de las fracciones. Educación Matemática, 25(2), 7-29. http://www.revista-educacion-matematica.com/descargas/Vol25-2.pdf

Creswell, J. W. (2012). Educational Research. Planning, Conducting and Evaluating Quantitative and Qualitative Research. Boston: Pearson.

Delacruz, G. C. (2018). Impact of Incentivizing the Use of Feedback on Learning and Performance in Educational Videogames. Hampton: NASA/Langley Research Center. https://files.eric. ed.gov/fulltext/ED530477.pdf

Donoso Vegas, R.; M. González Figueroa (2013). Herramientas no invasivas de observación en la evaluación de procesos de alfabetización informacional: Uso de Camstudio. Biblios, (52). https://doi.org/10.5195/biblios.2013.130

Flick, U. (2007). Introducción a la investigación cualitativa. Madrid: Morata.

García Gil, M. E. (2011). El video como herramienta de investigación. Una propuesta metodológica para la formación de profesionales en comunicación. Enlaces. Revista del CES Felipe II, (15). http://www.cesfelipesegundo.com/revista/articulos2011/MonicaGarcia.pdf 
García-Jiménez, E. (2015). La evaluación del aprendizaje: de la retroalimentación a la autorregulación. El papel de las tecnologías. RELIEVE: Revista Electrónica de Investigación y Evaluación Educativa, 21(2). http://dx.doi.org/10.7203/relieve.21.2.7546

González-Retana, J. F.; D. Eudave-Muñoz (2018). Conocimiento común del contenido del estudiante para profesor sobre fracciones y decimales. Educación Matemática, 30(2). https://doi. org/10.24844/em3002.05

Hattie, J.; M. Gan (2011). Instruction Based on Feedback. En Mayer, R.; P. Alexander (eds.). Handbook of Research on Learning and Instruction. Nueva York: Routledge, 249-271.

Hattie, J.; H. Timperley (2007). The Power of Feedback. Review of Educational Research, 7(1), 81112. https://doi.org/10.3102/003465430298487

Hinojoza-Rizo, S. E. (2001). Secuencia didáctica aplicada con los alumnos de $5^{\circ}$ grado de primaria en la resolución de adiciones de fracciones con diferente denominador. Guadalajara.

Huey-Min, W.; K. Bor-Chen; W. Su-Chen (2017). Computerized Dynamic Adaptive Tests with Immediately Individualized Feedback for Primary School Mathematics Learning. Journal of Educational Technology \& Society, 20(1), 61-72. https://www.jstor.org/stable/jeductechsoci.20.1.61

INEE (s.f.). Servicio Profesional Docente. http://servicioprofesionaldocente.sep.gob.mx/proceso/ -/desempenio

Kluger, A.; A. DeNisi (1996). The Effects of Feedback Interventions on Performance: A Historical Review, a Meta-analysis, and a Preliminary Feedback Intervention Theory. Psychological BuIletin, 119(2), 254-284. http://citeseerx.ist.psu.edu/viewdoc/download;jsessionid=8742A7A 9CBADEBFB774116443A47F51D?doi=10.1.1.461.6812\&rep=rep1\&type=pdf

Knoblauch, H.; R. Tuma (2011). Videography: An Interpretative Approach to Video-recorded Micro-social Interaction. En Margolis, E.; L. Pauwels (eds.). The Sage Handbook of Visual Research Methods. Londres: Sage, 414-430.

Kriscautzky, M.; E. Ferreiro (2017). Evaluar la confiabilidad de la información en Internet: cómo enfrentan el reto los nuevos lectores de 9 a 12 años. Perfiles Educativos, 40(159). 16-34. https://doi.org/10.22201/iisue.24486167e.2018.159.58306

Kvale, S. (2011). Las entrevistas en investigación cualitativa. Madrid: Morata.

MacDonald, V. A. (2015). The Application of Feedback in Secondary School Classrooms: Teaching and Learning in Applied Level Mathematics. Tesis doctoral. Toronto: Universidad de Toronto. https://tspace.library.utoronto.ca/bitstream/1807/69413/3/MacDonald Vincent A 201506 PhD thesis.pdf

Martínez-Rizo, F. (2013). Dificultades para implementar la evaluación formativa: Revisión de literatura. Perfiles Educativos, 35(139). 128-150. http://www.scielo.org.mx/pdf/peredu/ v35n139/v35n139a9.pdf

Diólo@os 
Marzano, R.; D. J. Pickering; J. E. Pollock (2001). Classroom Instruction that Works: Research-based Strategies for Increasing Student Achievement. Virginia: Association for Supervision and Curriculum Development.

Ontario Ministry of Education (2005). The Ontario Curriculum, Grades 9 and 10. Ontario: Queen's Printer for Ontario.

Pollock, J. E. (2012). How Feedback Leads to Engagement. Educational Leadership, 70(1). http:// www.ascd.org/publications/educational-leadership/sept12/vol70/num01/How-Feedback-Leads-to-Engagement.aspx

Rädiker, S.; U. Kuckartz (2020). Análisis de datos cualitativos con MaxQDA. Berlín: MaxQDA Press.

Ramos, S. L. (2010). Contextos CLIL para la formación inicial del profesorado de ciencias: análisis de la interacción desde una perspectiva sociocultural. Tesis doctoral. Barcelona.

Rathje, R. J. (2018). A Qualitative Case Study of Mathematics Teachers' Formative Assessment Feedback. Tesis doctoral. Minneapolis: Walden University. https://scholarworks.waldenu.edu/ cgi/viewcontent.cgi?article $=7213 \&$ context $=$ dissertations

Ravela, P.; B. Picaroni, G. Loureiro (2017). ¿Cómo mejorar la evaluación en el aula? México: Magro Editores.

Saldaña, J. (2009). The Coding Manual for Qualitative Researches. Londres: Sage.

SEP (2012). La comunicación de los logros de aprendizaje de los alumnos desde el enfoque formativo. México: SEP.

SEP (s.f.). Dirección General de Educación Superior para Profesionales de la Educación. https://www. dgespe.sep.gob.mx/

Shute, V. J. (2008). Focus on Formative Feedback. Review of Educational Research, 153-189.

Souter, D. H. (2009). The Nature of Feedback Provided to Elementary Students by Teachers in Schools where Grading and Reporting are Standards-based. Atlanta: Universidad de Georgia. https:// scholarworks.gsu.edu/eps diss/62/

Stake, R. E. (1999). Investigación con estudio de casos. Madrid: Morata.

Temple Adger, C. (2001). Discourse in Educational Settings. The Handbook of Discourse Analysis. Gran Bretaña: Blackwell Publishers.

Wiliam, D. (1999). Formative Assessment in Mathematics-Part 2: Feedback. Equals: Mathematics and Special Education Needs, 5(3), 8-11. https://discovery.ucl.ac.uk/id/eprint/10001148/1/ Wiliam1999Formativepart2 8.pdf

Wiliam, D. (2012). Feedback: Part of the System. Educational Leadership, 70(7), 31-34.

Yin, R. K. (2018). Case Study Research and Applications: Design and Methods. Los Ángeles: Sage. 